\title{
ON THE CHEMODYNAMICS OF NGC 6888
}

\author{
C. ESTEBAN and J.M. VILCHEZ \\ Instituto de Astrofísica de Canarias \\ E-38200, La Laguna, Tenerife, Spain
}

\begin{abstract}
We present preliminary results on an extensive spectroscopical study of the WR Ring nebula NGC 6888. The observations combine high spatial $(1.5 \mathrm{arcsec} / \mathrm{pixel})$ and spectral resolution (30 to $50 \mathrm{~km} \mathrm{~s}^{-1}$ ) covering most of the optical range $-\lambda \lambda 3600$ to $6800 \AA-$ at 3 different slit positions along the major axis of the nebula. The spectra of the central parts give an emission system with three different velocities: a) $\mathrm{V}_{L S R}=-64 \mathrm{~km} \mathrm{~s}^{-1}$, b) $\mathrm{V}_{L S R}=+18 \mathrm{~km} \mathrm{~s}^{-1}$, and c) $V_{L S R}=+78 \mathrm{~km} \mathrm{~s}^{-1}$. Assuming that NGC 6888 is in fact an expanding bubble of gas, as demonstrated by Marston and Meaburn (1988), we can identify components a) and c) as those moving towards us and receeding parts of the shell, while b) could be related to the ambient interstellar ionized gas outside the bubble. We have isolated the spectrum for each component in order to analyse the ionization structure and excitation mechanism, as well as to derive their physical conditions and chemical abundances. The use of diagnostic diagrams indicates that the nebula is basically photoionized, without any significant contribution of shock excitation in the zones studied. The ambient interstellar component shows a spectrum typical of an $\mathrm{H}$ II region with nearly solar abundances. In the case of the bubble components, their spectra produce [N II] $/ \mathrm{H} \alpha$ line ratios which are outside the HII region box, and entering the extended planetary nebulae locus, suggesting a contribution of ejected nitrogen in the bubble. This fact is evident from the abundance analysis we have performed, finding that, with respect to the values quoted for the ambient gas, the $\mathrm{O} / \mathrm{H}$ is deficient by a factor $4, \mathrm{~N} / \mathrm{H}$ is 2.5 times higher, and helium is enhanced by a factor 2 . These quoted values clearly indicate that a substantial fraction of the gas in the bubble is processed material ejected from the central massive star.
\end{abstract}

K. A. van der Hucht and B. Hidayat (eds.),

Wolf-Rayet Stars and Interrelations with Other Massive Stars in Galaxies, 422.

(C) 1991 IAU. Printed in the Netherlands. 